\title{
Argon gas: a potential neuroprotectant and promising medical therapy
}

\author{
Derek S Nowrangi ${ }^{1}$, Jiping Tang ${ }^{1 *}$ and John H Zhang ${ }^{1,2^{*}}$
}

\begin{abstract}
Argon is a noble gas element that has demonstrated narcotic and protective abilities that may prove useful in the medical field. The earliest records of argon gas have exposed its ability to exhibit narcotic symptoms at hyperbaric pressures greater than 10 atmospheres with more recent evidence seeking to display argon as a potential neuroprotective agent. The high availability and low cost of argon provide a distinct advantage over using similarly acting treatments such as xenon gas. Argon gas treatments in models of brain injury such as in vitro Oxygen-Glucose-Deprivation (OGD) and Traumatic Brain Injury (TBI), as well as in vivo Middle Cerebral Artery Occlusion (MCAO) have largely demonstrated positive neuroprotective behavior. On the other hand, some warning has been made to potential negative effects of argon treatments in cases of ischemic brain injury, where increases of damage in the sub-cortical region of the brain have been uncovered. Further support for argon use in the medical field has been demonstrated in its use in combination with tPA, its ability as an organoprotectant, and its surgical applications. This review seeks to summarize the history and development of argon gas use in medical research as mainly a neuroprotective agent, to summarize the mechanisms associated with its biological effects, and to elucidate its future potential.
\end{abstract}

Keywords: Argon, Xenon, Noble gas, Inert gas, Narcosis, Anesthesia, Neuroprotection, Organoprotection, Ischemia, Brain injury, Oxygen-Glucose deprivation, Traumatic brain injury, Middle cerebral artery occlusion, Tissue plasminogen activation, Argon plasma coagulation

\section{Introduction}

Argon gas is considered a small noble gas element that has been applied in a number of fields. It has been generally classified as a nonreactive or inert gas providing a view that it does not contain any biologically active characteristics. In fact, argon has demonstrated characteristics such as narcosis at hyperbaric pressures and more recently neuroprotective and organoprotective behaviors. Pharmaceutical drugs and surgical interventions have been widely accepted and commonly used methods of clinical treatments for a variety of applications such as the neuroprotection [1-4]. However, the high cost and development of new drugs and surgical techniques have exposed a

\footnotetext{
* Correspondence: jtang@llu.edu; johnzhang3910@yahoo.com

'Department of Physiology, Loma Linda University School of Medicine, Risley Hall, Room 223, Loma Linda, CA 92354, USA

${ }^{2}$ Department of Neurosurgery, Loma Linda University School of Medicine,
} Loma Linda, CA, USA

\section{() Biomed Central}

need for new and easy to administer treatments. The application of argon as a medical gas presents a possible relief to this search and provides distinct advantages.

This review seeks to show the development of argon in the medical field with an emphasis on its ability as a neuroprotectant. In order to provide a foundation of argons role in the medical field, a summary of its discovery and characteristics will be first provided. We will then discuss the evidence suggesting the affirmative ability of argon as a neuroprotectant and highlight some evidence suggesting otherwise. In order to better understand how argon provides its biological actions, a review and discussion of the possible mechanisms of receptor interactions and apoptotic cellular pathways will be provided. Finally, we will consider the future of argon as a clinical therapy and other potential applications in the medical field. 


\section{History and characteristics of argon}

In 1785, Henry Cavendish isolated atmospheric gases in an attempt to characterize and quantify the substituents. In an attempt to combust the samples to find their reactivity profile, a small constituent of isolated gas samples believed to be nitrogen did not give the expected reaction and remained unchanged. After further analysis, it was concluded that the nonreactive nature of this gas sample was considered to be an error due to contamination [5]. The results were brought to attention again in 1894 when Lord Rayleigh and William Ramsay's experimentation on atmospheric gases found that a similar amount of unreactive gas contained a unique set of characteristics. They confirmed that the unreactive gas was observed by Henry Cavendish and classified it to be a new element "Argon" [6].

Argon belongs to a family of elements located on the final column of the periodic table of elements termed "noble gases" which include the elements helium, neon, argon, krypton, xenon, and radon. The full electron valence shell of these elements prevents the formation covalent bonds and finding these gases in compound form remains a rare occurrence [7]. The inability to form strong covalent bonds with other elements develops an identity for the gases to be unreactive, grouping these gases under another term: inert gases. Though the term "inert gas" implies these gases have no active characteristics, several instances have been noted in which these gases are able to produce physical and biological effects [8]. The biological activity of argon can be attributed to its atomic structure interactions with enzymes and receptors. Though argon is unable to form strong covalent bonds to produce chemical reactions, it does have the capacity to interact with enzymes and receptors through chargeinduced dipole and van der Waals interactions as a stabilizing component key in relating argon's ability as an anesthetic and neuroprotectant $[9,10]$.

Due to the similar structures and reactive natures of argon and xenon, a propensity to compare the two elements has appeared in a number of reports [11-13]. Xenon is one of the earliest and most widely investigated of the noble gases and is regarded as a potent anesthetic and a convincing neuroprotectant [14-17]. However, the limited availability $(0.09 \mathrm{ppm})$ and high cost of xenon has prevented it from becoming a commonly used therapy option. The property of xenon as an inert gas has opened speculation of the other noble gases as possible alternatives with several of the noble gases revealing potentials in the medical field. In particular, argon displays attributes that position it as a likely alternative to xenon especially in the field of neuroprotection. Furthermore, the reasonably high availability $(9340 \mathrm{ppm})$ of argon in the atmosphere allows for a low difficulty in obtaining the gas and makes argon relatively more cost effective as compared to xenon [18].

\section{Effects of argon as a medical gas Argon as a narcotic agent}

The first biological effect of argon gas can be found as a description of its narcotic capabilities represented in a study pertaining to the high-pressure effects of naturally occurring gases during deep sea diving. Previous observations noted that under high pressure with normal respiratory air, divers begin to develop narcotic symptoms of slowed mental cognition and psychological instability [19-21]. After isolating the gases, it was surmised that argon gas produces a strong narcotic effect at high pressures (>10 atm) as compared to helium and nitrogen, while xenon is able to produce narcotic symptoms at atmospheric pressure [22]. It was also theorized that the narcotic effects argon exerts is being created in a physical rather than a chemical manner due to its characteristic as inert gas lacking chemical reactions in the body [23].

The mechanism in which argon displays its anesthetic ability has been suggested to be from the stimulation of $\gamma$-aminobutyric acid type-A receptors $\left(\mathrm{GABA}_{\mathrm{A}} \mathrm{R}\right)$ [24]. Though argons involvement has yet to be confirmed, other anesthetic gases such as nitrogen have also been suggested to stimulate the $\mathrm{GABA}_{\mathrm{A}} \mathrm{R}$ as well [25]. Of further interest, other anesthetic gases such as xenon and nitrous oxide are described to antagonize N-methyl-D-aspartate receptors (NMDAR) to promote their narcotic effects $[26,27]$. The antagonism of NMDAR remains a plausible method for argon-induced anesthesia, but has yet to be established and is still under investigation. Additionally, dopamine release has been connected to both the activity of $\mathrm{GABA}_{\mathrm{A}} \mathrm{R}$ and NMDAR, and a decrease in dopamine activity in the brain would promote a narcotic effect $[28,29]$. Argon gas treatments to rats found that levels of extracellular striatal dopamine are reduced at pressures exceeding $2.5 \mathrm{MPa}$ (approximately $10 \mathrm{~atm}$ ), suggesting a neurochemical method of which argon may present its anesthetic effects [30].

A number of gases including the noble gas family exhibit anesthetic tendencies, but greatly differ in the criteria required for their narcotic abilities to manifest [31]. Argon has been described as being an anesthetic agent, but is only able to exert these effects under hyperbaric pressures. Xenon has also been portrayed as a potent anesthetic, but manifests its narcotic effects at normobaric pressures. The noble gases helium and neon lack an observable anesthetic effect for reasons still under investigation [31,32]. The capacity of argon and xenon to 
produce anesthetic effects at different pressures may signify an important difference when considering their treatment potential in the medical field.

\section{Ischemic neuroprotective models}

Of the potential uses argon in the medical field, studies pertaining to its ability as a neuroprotective agent have been most prominently examined. Investigations of neuroprotection seek to improve recovery of motor and behavioral functions of patients that have experienced neurological damage in cases such as but not limited to physical trauma [33,34]. Argon neuroprotective studies have largely been examined models of ischemic brain injuries in which the deprivation of essential nutrients such as glucose and oxygen to the brain may damage tissues and activate inflammatory and apoptotic pathways in surrounding tissues leading to neuronal death [35-39]. Oxygen-Glucose Deprived (OGD) environments, Traumatic Brain Injury (TBI), and the Middle Cerebral Artery Occlusion (MCAO) models are highly accepted methods of establishing ischemic brain injury treatments in rodent models and are the common methods of which argon neuroprotection treatments have been examined (Figure 1). An OGD model places a tissue of interest in a medium depriving the tissue of oxygen and glucose to simulate ischemic conditions in vitro [40]. The TBI model is a physical method of applied blunt force to the skull resulting in inflammatory and ischemic conditions with treatments possible in vitro and in vivo [41,42]. The MCAO model is considered an in vivo method of
(A)

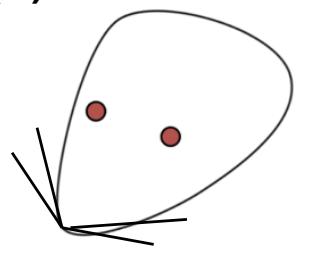

(B)

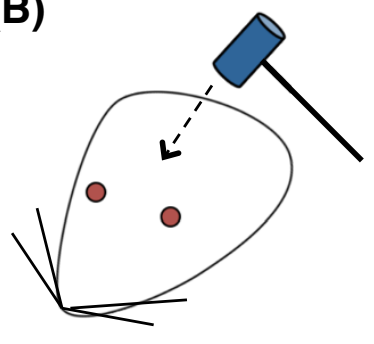

(C)

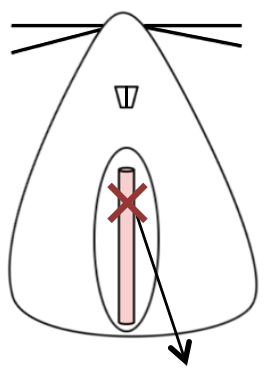

Middle Cerebral Artery Ligation
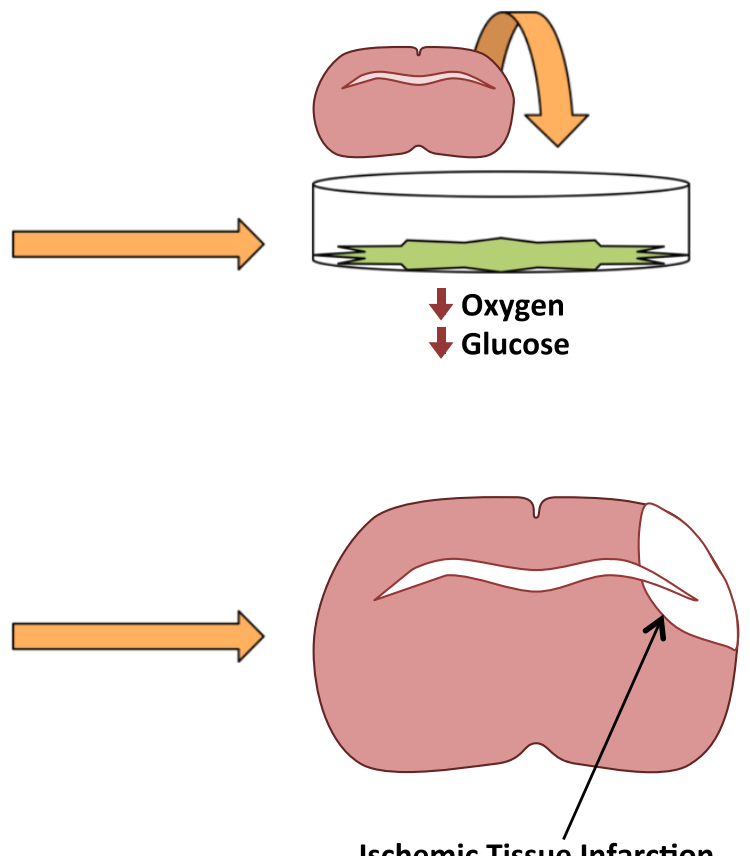

Ischemic Tissue Infarction

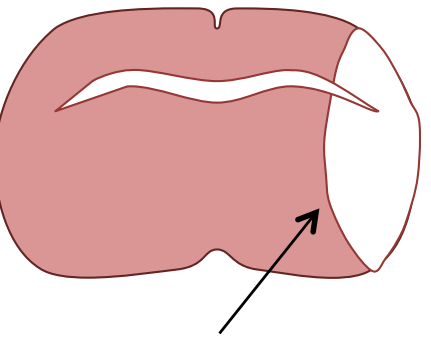

Ischemic Tissue Infarction

Figure 1 Commonly used ischemic models of neuroprotection. (A) An OGD model of neuroprotection places brain tissue into a medium that deprives it of oxygen and glucose in vitro. (B) The TBI model uses an apparatus to cause a forceful impact on the brain and results in ischemic tissue damage with treatments possible in vivo or in vitro. (C) The MCAO model ligates the middle cerebral artery to produce an ischemic infarction with treatments examined in vivo. 
ligating the middle cerebral artery to simulate ischemic arterial blockage followed by reperfusion [43].

\section{Argon neuroprotection in vitro}

A study by Jawad and colleagues aimed to compare the neuroprotective ability of the noble gases helium, neon, argon, and krypton to xenon in an Oxygen-Glucose Deprived (OGD) environment. Fetal mice cerebral cortices were exposed to an OGD medium and then treated to the noble gases $\left(75 \%\right.$ noble gas, $20 \% \mathrm{O}_{2}$, and $\left.5 \% \mathrm{CO}_{2}\right)$. It was observed that argon and xenon improved neuronal survival while helium, neon, and krypton failed to significantly reduce neuronal death. Additionally, they observed that administering the gases without an OGD environment, is able to improve neuronal survival as compared to a control environment $\left(75 \% \mathrm{~N}_{2}\right.$ instead of noble gas) [18].

Loetscher and colleagues examined both models of OGD and TBI in vitro and found that all concentrations of the argon-oxygen gas mixtures tested $(25-74 \% \mathrm{Ar}$, $21 \% \mathrm{O}_{2}, 5 \% \mathrm{CO}_{2}, \mathrm{~N}_{2}$ the rest) were effective in reducing cellular trauma injury to hippocampal brain slices of mice pups in both models. It was also noted that in the TBI model 50\% argon exhibited the greatest reduction in injury but a concentration of $74 \%$ argon greatly attenuated this reduction in damage signifying a dose-dependent relationship of argon gas treatments. Additionally, it was demonstrated that delayed argon administrations given up to 3 hours after the insult were effective in reducing tissue damage [44]. Similarly, a study by Harris and colleagues found that argon (50\% atm) and xenon (50\% atm) gas are able to greatly attenuate the amount of injury up to 72 hours after TBI examined in vitro. The author's note that though argon was able to reduce the total injury significantly, it was not as great of a reduction as found by Loetscher and colleagues [45].

\section{Argon neuroprotection in vivo}

An in vivo examination by Ryang and colleagues using the MCAO model treated adult rats with 50\% argon $\left(50 \% \mathrm{O}_{2}\right) 1$ hour following reperfusion of the middle cerebral artery. Reversals in tissue damage and a reduction in the edema-infarct volumes were observed up to 24 hours after reperfusion of the artery. The argon treated rats also demonstrated improved neurological function suggesting greater levels recovery and supporting the positive neuroprotective ability of argon. Furthermore, no significant differences of heart rate, blood pressure, and blood-gas measurements were monitored after argon inhalation signifying that normal physiological parameters were not disturbed [46].

Further supporting evidence for argon gas neuroprotection can be found in a study by Zhuang and colleagues aiming to compare noble gases treatments of helium, argon, and xenon to a control of nitrogen in a model of neonatal hypoxia-induced brain injury. Gas treatments $\left(70 \% \mathrm{Ar} / \mathrm{He} / \mathrm{Xe}\right.$ and $\left.30 \% \mathrm{O}_{2}\right)$ were given 2 hours after the hypoxic insult in which the right common carotid artery was ligated. They observed that cell morphology in the hippocampus right hemisphere was significantly restored for all treatment groups, but that argon was able to restore cell viability to the greatest extent. It was also noted that argon and xenon treatment groups displayed reductions of infarction sizes and that neurocognitive behavior results showed improvement for all gases [47].

Patients suffering a cardiac arrest may exhibit neurological damage by consequence of global brain ischemia and deregulation of elements such as coagulation and inflammatory factors [48]. Post-cardiac arrest, the sustained neurological damage may provide complications in the survival and recovery. In an effort to remedy this situation, Brücken and colleagues examined the effects argon gas provided after cardiac arrest in rats. The treatments were applied 1 hour after cardiac arrest resuscitation using $70 \%$ argon $\left(30 \% \mathrm{O}_{2}\right)$. It was found that argon inhalation improved neurological scores with fewer damaged neurons observed in the cortex and hippocampal regions [49]. Similarly, Ristagno and colleagues found that treatments of $70 \%$ argon $\left(30 \% \mathrm{O}_{2}\right)$ for 4 hours following porcine cardiac arrest resuscitation resulted in significantly improved neurological scores up to 72 hours after the injury [50].

\section{Negative neuroprotective effect}

Though a number of ischemic brain injury studies have suggested a beneficial neuroprotective outcome due to argon gas exposure, a study by David and colleagues found that there remains a possibility for adverse consequences. A three-part examination found that positive neuroprotection was noted in cases of OGD and Nmethyl-D-aspartate (NMDA) induced neuronal death, but negative effects were found in a model of MCAO. An in vivo treatment of $50 \%$ argon $\left(50 \% \mathrm{O}_{2}\right)$ was applied 2 hours after a MCAO-induced injury in adult rats. Though the total brain damage was decreased, it was also found that argon increased the amount of damage found in the sub-cortex region with no improvements of behavior or motor functions. This is the first study to reveal a detrimental outcome of argon gas treatments in a model of neuroprotection [51].

The examination of the OGD-induced environment measured lactate dehydrogenase (LDH) as a marker of neuronal injury and found that argon-oxygen gas exposure (37.5\% to $75 \%$ argon) decreased neuronal injury with the greatest reduction in damage found 3 hours after the insult. Additionally, NMDA injections were used to stimulate neuronal death with treatments of argon-oxygen gas 
(15-75\% argon) given 1 hour after injection. Consistent with previous studies, treatments of 50\% argon showed the maximal reduction of neuronal death in both the OGD and NMDA-induced cytotoxic environments. Though improvements were seen in the OGD environment and NMDAinduced cytotoxic models, the authors assess that argon gas would not be a suitable post-ischemic neuroprotective treatment for clinical use due to the negative outcome found in the MCAO examination, but also suggest that argon may be a useful neuroprotectant for other brain injuries such as TBI.

\section{Mechanisms}

\section{NMDAR mediated neuroprotection}

As a neuroprotectant, little is understood about argons interactions to receptors and enzymes or the cellular pathways involved after its initial interactions, though there have been hints as to the involvement of NMDAR and $G_{A B A} R$. Both NMDAR and GABA $_{A} R$ are widely accepted to be involved in a number of cell survival pathways and are also considered to be major targets of a number of anesthetics [52-55]. NMDAR are largely stimulated by glutamate and are considered to be excitatory neuronal receptors, while $\mathrm{GABA}_{\mathrm{A}} \mathrm{R}$ are largely stimulated by $\gamma$-aminobutyric acid (GABA) and are considered to be inhibitory neuronal receptors $[26,56]$. It should also be considered that an increase of activity of one receptor type could result in lowering the activity of the other due to their similar but opposite involvement in their excitatory actions.

Previous reports have theorized that NMDAR interactions may be implicated in the mechanism of argon gas neuroprotection. An intrinsic pro-apoptotic mitochondrial pathway is activated in consequence of increased glutamate release and stimulation of NMDAR. The overstimulation of NMDAR produces a large influx of intracellular $\mathrm{Ca}^{2+}$, which is known to be a major cause of cytotoxic neuronal death [57-61]. The influx of $\mathrm{Ca}^{2+}$ triggers a rise in the pro-apoptotic Bax protein that competes with the anti-apoptotic signaling of Bcl-2 and $\mathrm{Bcl}-\mathrm{xL}$ proteins $[57,62,63]$. Zhuang and colleagues found that the argon treated groups promoted an increased expression of the pro-survival protein $\mathrm{Bcl}-2$ while having no effect on Bax or Bcl-xL expression in neuronal cells [47]. Furthermore, the attenuation of NMDA induced neuronal damage by argon gas observed by David and colleagues offers additional conjecture of NMDAR involvement [51].

Conversely, Harris and colleagues provide evidence that NMDAR are likely not involved in the neuroprotective activity of argon. Using glycine as a competitive inhibitor of NMDAR, no changes in argons neuroprotective behavior were discovered suggesting another mechanism in which argon activity is mediated. In addition, they also found through electrophysiological methods that the activity of
TREK-1 potassium channels is not affected with argon application. Though the study didn't expand on the cellular mechanisms of the neuroprotective behavior seen, it provides a clear representation of how NMDAR do not seem to be involved in argons neuroprotective behavior [45].

\section{$\mathrm{GABA}_{\mathrm{a}} \mathrm{R}$ mediated neuroprotection}

It can also be proposed that $\mathrm{GABA}_{\mathrm{A}} \mathrm{R}$ may play a role in producing the neuroprotective effects of argon rather than NMDAR. Argon has been suggested to change $\mathrm{GABA}_{\mathrm{A}} \mathrm{R}$ activity by binding to multiple discrete sites on the receptor [24]. However, this observation was suggested in relation to argons narcotic properties with no relationship to its neuroprotective properties being made. Several examinations of other drugs have observed that the stimulation of $\mathrm{GABA}_{\mathrm{A}} \mathrm{R}$ has the ability produce neuroprotective results [64-66]. Of important consideration is that the involvement of $\mathrm{GABA}_{\mathrm{A}} \mathrm{R}$ to argon signaling is in relation to its narcotic properties that are displayed only at hyperbaric pressures, while neuroprotection studies of argon are largely done at normobaric pressure.

\section{Mek-erk 1/2 pathway involvement}

The neuroprotective behavior of argon may not be receptor mediated but may be the result of direct pathway participation. Fahlenkamp and colleagues noticed that argon treatments to cells in cultures increased expression of extracellular signal regulated kinases (ERK) $1 / 2$ in neurons, microglia, and astrocyte cells. ERK $1 / 2$ is a highly ubiquitous protein associated with a number of cellular pathways such as inflammatory and cell survival pathways depending on the methods of activation. It has been demonstrated that ERK $1 / 2$ is largely regarded as a pro-death marker of neuronal cells, but through methods of transient stimulation can also be part of a pro-survival pathway [67]. Though these results did not investigate outcome results associated with the increase of ERK $1 / 2$, it can be proposed that argon may be connected to the mitogen activated protein kinase (MAPK) kinase (MEK)-ERK $1 / 2$ pathway in neuronal cells. Measurements found that argon does not change the level of phosphotyrosine phosphatases suggesting that an indirect cellular pathway is not stimulated. They suggest that because of the varied nature and independency from the cellular background of the MEK-ERK $1 / 2$ pathway, argon may directly stimulate the pathway to assert its neuroprotective effects rather than through receptor mediation [68] (Figure 2).

\section{Future outlook}

The use of argon gas for neuroprotective medical applications is a recent endeavor that has not expanded into clinical examinations as of yet. However, David and colleagues provided an examination on argons effects in 


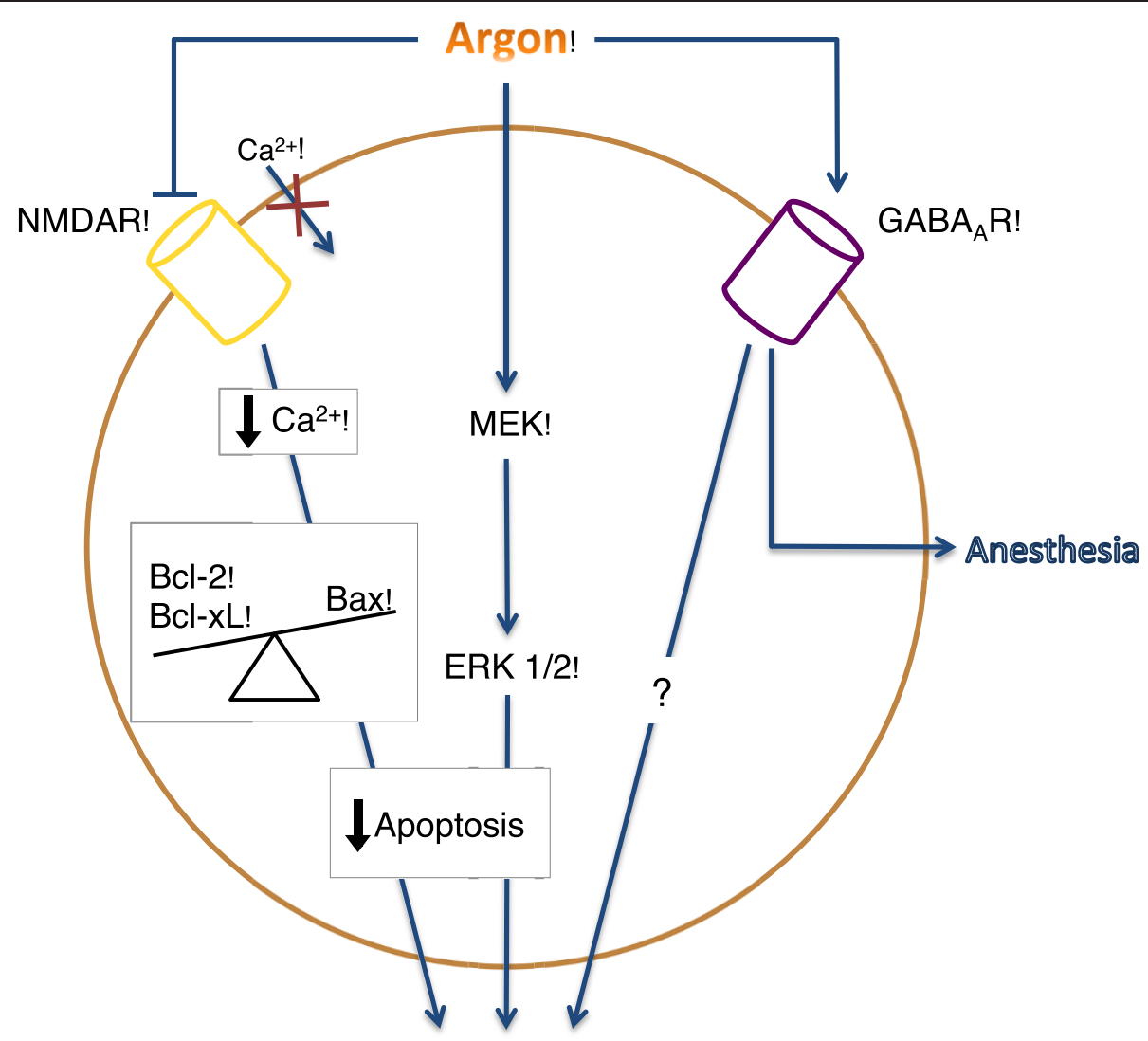

\section{NEUROPROTECTION!}

Figure $\mathbf{2}$ Theorized argon neuroprotective pathways. Suggested pathways still under investigation that may contribute to the neuroprotective effects of argon gas treatments in neuron cells include: NMDA receptor inhibition, direct stimulation of the MEK and ERK $1 / 2$ anti-apoptotic pathway, and stimulation $\mathrm{GABA}_{\mathrm{A}}$ receptor.

combination with tissue plasminogen activator (tPA) that can be regarded as a indicator that an interest in its clinical application exists. In cases of acute ischemic stroke, the current and only approved method of clinical therapy has been the administration of tPA. At levels below $50 \%$ argon gas $(25 \%$ oxygen and the rest nitrogen to complete mixture), argon decreased the catalytic and thrombolytic efficiency of tPA, but increased them at levels above $50 \%$ argon. These results seem to promote that argon gas may enhance the efficiency of tPA treatments and may foster support for use in clinical examination [69].

Besides argons use as a neuroprotectant, argon gas has also demonstrated organoprotectant traits. Irani and colleagues found that kidney recovery after storage with argon gas was improved as compared with mediums of xenon, nitrogen, or air. It was assessed that argon gas may provide a potential organoprotecive environment for organ transplants by preserving the quality and function of the kidney [70]. In addition, another study found that argon gas is able to protect the myocardium to infarction in cases of coronary artery occlusion and reperfusion. This study also examined the role of ERK $1 / 2$ signaling, but did not find any changes in response to argon gas treatments [71]. It has also been found that argon and xenon $\left(75 \% \mathrm{Ar} / \mathrm{Xe}, 20 \% \mathrm{O}_{2}\right.$, and $\left.5 \% \mathrm{CO}_{2}\right)$ are able to limit apoptotic cell death when given up to 16 hours after inducing apoptosis to osteosarcoma cells in culture [72].

Another use of argon in the medical field has been as a surgical tool. Argon Plasma Coagulation (APC) is a non-contact technique that uses high frequency stimulation of argon plasma to cauterize surrounding tissues and prevent bleeding through coagulation around surgical sites. APC is an example of an early use of argon gas in a medical environment demonstrating an ability to effectively limit tissue damage as seen in surgical cases including skin, gastrointestinal, and neurosurgeries [73-77]. The positive feedback from the use APC has allowed for speculation on its aptitude as a suitable replacement for standardized coagulation techniques such as bipolar coagulation [78].

\section{Conclusion}

The development of argon in medical research has originated from its ability as a narcotic agent to a gas with 
potential protective properties. The greater availability and low cost of argon provide a distinct advantage over xenon while the difference in the pressure required to exhibit narcotic properties allows for a variety of clinical applications not available to xenon. Ischemic brain injury models tend to show that argon is able to produce positive neuroprotective effects, though there also exists possible negative effects of argon gas therapies. Furthermore, the mechanisms in which argon exerts its neuroprotective behavior are poorly understood. Therefore, it can be evoked that an inadequate amount of data exists to correctly assess argons neuroprotective capability. Though studies investigating argon gas use in clinical therapies have yet to be examined, support for its use is evident through the combinational report with tPA. In addition, argon has garnered support as an organoprotectant and has shown progress as a surgical tool. Though much is still unknown about the effects and mechanisms of argon, a number of promising signs have been given to its future in the medical field with an emphasis found in its neuroprotective ability.

\section{Abbreviations}

LDH: Lactate dehydrogenase; OGD: Oxygen glucose deprivation; TBI: Traumatic brain injury; MCAO: Middle cerebral artery occlusion; NMDA: N-Methyl-D-Aspartate; NMDAR: N-Methyl-D-Aspartate receptor; GABA: $\gamma$-Aminobutyric ccid; $G A B A_{A} R$ : $\gamma$-Aminobutyric acid type-A receptor; tPA: Tissue plasminogen activator; APC: Argon plasma coagulation.

\section{Competing interests}

The authors declare that they have no competing interests.

\section{Authors' contributions}

DSN - Role included reviewing manuscripts, review design, manuscript preparation, and manuscript editing. JT - Role included manuscript proof reading. JZ - Role included review design and manuscript proof reading. All authors read and approved the final manuscript.

\section{Acknowledgements}

We thank Damon Klebe and Jerry Flores for contributions to the conception and design.

Received: 4 October 2013 Accepted: 11 February 2014 Published: 17 February 2014

\section{References}

1. De Keyser J, Sulter G, Luiten PG: Clinical trials with neuroprotective drugs in acute ischaemic stroke: are we doing the right thing? Trends Neurosci 1999, 22(12):535-540.

2. Ito $\mathrm{H}$, et al: Neuroprotective properties of propofol and midazolam, but not pentobarbital, on neuronal damage induced by forebrain ischemia, based on the GABAA receptors. Acta Anaesthesiol Scand 1999, 43(2):153-162

3. Bilotta F, et al: Pharmacological perioperative brain neuroprotection: a qualitative review of randomized clinical trials. Br J Anaesth 2013, 110(Suppl 1):i113-i120

4. Tator $\mathrm{CH}$, et al: Translational potential of preclinical trials of neuroprotection through pharmacotherapy for spinal cord injury. J Neurosurg Spine 2012, 17(1 Suppl):157-229.

5. Cavendish H: Experiments on Air. By Henry Cavendish, Esq. F. R. S. \& S. A. Philos Trans R Soc Lond 1784, 74:119-153.

6. Rayleigh L, Ramsay W: Argon, a new constituent of the atmosphere. Proc R Soc Lond 1894, 57(340-346):265-287.
7. Christe KO: Bartlett's discovery of noble gas fluorides, a milestone in chemical history. Chem Commun (Camb) 2013, 49(41):4588-4590.

8. Ruzicka J, et al: Biological effects of noble gases. Physiol Res 2007, 56(Suppl 1):S39-S44.

9. Trudell JR, Koblin DD, Eger El 2nd: A molecular description of how noble gases and nitrogen bind to a model site of anesthetic action. Anesth Analg 1998, 87(2):411-418.

10. Quillin ML, et al: Size versus polarizability in protein-ligand interactions: binding of noble gases within engineered cavities in phage T4 lysozyme. J Mol Biol 2000, 302(4):955-977.

11. Gudmundsson JT, Lieberman MA: Ar + and Xe + velocities near the presheath-sheath boundary in an Ar/Xe discharge. Phys Rev Lett 2011, 107(4):045002

12. Schiwietz $\mathrm{G}$, et al: Evidence for an ultrafast breakdown of the $\mathrm{BeO}$ band structure due to swift argon and xenon ions. Phys Rev Lett 2010, 105(18):187603.

13. Kyrychenko A, Waluk J: Molecular dynamics simulations of matrix deposition. III. Site structure analysis for porphycene in argon and xenon. J Chem Phys 2005, 123(6):64706.

14. Ma D, et al: Neuroprotective and neurotoxic properties of the 'inert' gas, xenon. Br J Anaesth 2002, 89(5):739-746.

15. Preckel $B$, et al: Molecular mechanisms transducing the anesthetic, analgesic, and organ-protective actions of xenon. Anesthesiology 2006, 105(1):187-197.

16. Derwall $M$, et al: Xenon: recent developments and future perspectives. Minerva Anestesiol 2009, 75(1-2):37-45.

17. Franks NP, et al: How does xenon produce anaesthesia? Nature 1998, 396(6709):324.

18. Jawad N, et al: Neuroprotection (and lack of neuroprotection) afforded by a series of noble gases in an in vitro model of neuronal injury. Neurosci Lett 2009, 460(3):232-236.

19. Behnke AR, Thompson RM, Motley EP: The psychologic effects from breathing air at 4 atmospheres pressure. Am J Physiol Legacy Content 1935, 112(3):554-558.

20. Dudley SF: Some atmospheric hazards encountered in naval life: (united services section). Proc R Soc Med 1935, 28(9):1283-1292.

21. Haldane JBS: Human life and death at high pressures. Nature 1941 148:458-460.

22. Lawrence JH, et al: Preliminary observations on the narcotic effect of xenon with a review of values for solubilities of gases in water and oils. J Physiol 1946, 105(3):197-204.

23. Behnke AR, Yarbrough OD: Respiratory resistance, oil-water solubility, and mental effects of argon, compared with helium and nitrogen. Am J Physiol Legacy Content 1939, 126(2):409-415.

24. Abraini JH, et al: Gamma-aminobutyric acid neuropharmacological investigations on narcosis produced by nitrogen, argon, or nitrous oxide. Anesth Analg 2003, 96(3):746-749. table of contents.

25. Rostain JC, Balon N: Recent neurochemical basis of inert gas narcosis and pressure effects. Undersea Hyperb Med 2006, 33(3):197-204.

26. Franks NP, Lieb WR: Molecular and cellular mechanisms of general anaesthesia. Nature 1994, 367(6464):607-614.

27. Jevtovic-Todorovic $V$, et al: Nitrous oxide (laughing gas) is an NMDA antagonist, neuroprotectant and neurotoxin. Nat Med 1998, 4(4):460-463.

28. Luo R, Partridge JG, Vicini S: Distinct roles of synaptic and extrasynaptic GABAAreceptors in striatal inhibition dynamics. Front Neural Circuits 2013, 7:186.

29. Ladepeche L, Dupuis JP, Groc L: Surface trafficking of NMDA receptors: Gathering from a partner to another. Semin Cell Dev Biol 2013. doi: 10.1016/.jsemcdb.2013.10.005

30. Balon N, et al: Opposing effects of narcotic gases and pressure on the striatal dopamine release in rats. Brain Res 2002, 947(2):218-24.

31. Koblin DD, et al: Minimum alveolar concentrations of noble gases, nitrogen, and sulfur hexafluoride in rats: helium and neon as nonimmobilizers (nonanesthetics). Anesth Analg 1998, 87(2):419-24.

32. Fowler B, Ackles KN, Porlier G: Effects of inert gas narcosis on behavior-a critical review. Undersea Biomed Res 1985, 12(4):369-402.

33. McConeghy KW, et al: A review of neuroprotection pharmacology and therapies in patients with acute traumatic brain injury. CNS Drugs 2012, 26(7):613-36.

34. Liu R, et al: Neuroprotection targeting ischemic penumbra and beyond for the treatment of ischemic stroke. Neurol Res 2012, 34(4):331-7.

35. Russo $R$, et al: In search of new targets for retinal neuroprotection: is there a role for autophagy? Curr Opin Pharmacol 2013, 13(1):72-7. 
36. Zhang $\mathrm{M}$, et al: Emerging roles of $\mathrm{Nrf2}$ and phase II antioxidant enzymes in neuroprotection. Prog Neurobiol 2013, 100:30-47.

37. Neal JW, Gasque P: How does the brain limit the severity of inflammation and tissue injury during bacterial meningitis? J Neuropathol Exp Neurol 2013, 72(5):370-85.

38. Lee JM, Zipfel GJ, Choi DW: The changing landscape of ischaemic brain injury mechanisms. Nature 1999, 399(6738 Suppl):A7-14.

39. Durukan A, Tatlisumak T: Acute ischemic stroke: overview of major experimental rodent models, pathophysiology, and therapy of focal cerebral ischemia. Pharmacol Biochem Behav 2007, 87(1):179-97.

40. Strasser U, Fischer G: Protection from neuronal damage induced by combined oxygen and glucose deprivation in organotypic hippocampal cultures by glutamate receptor antagonists. Brain Res 1995, 687(1-2):167-74.

41. Prins $M$, et al: The pathophysiology of traumatic brain injury at a glance. Dis Model Mech 2013, 6(6):1307-1315.

42. Namjoshi DR, et al: Towards clinical management of traumatic brain injury: a review of models and mechanisms from a biomechanical perspective. Dis Model Mech 2013, 6(6):1325-1338.

43. Liu F, McCullough LD: Middle cerebral artery occlusion model in rodents: methods and potential pitfalls. J Biomed Biotechnol 2011, 2011:464701.

44. Loetscher PD, et al: Argon: neuroprotection in in vitro models of cerebra ischemia and traumatic brain injury. Crit Care 2009, 13(6):R206.

45. Harris K, et al: Neuroprotection against Traumatic Brain Injury by Xenon, but Not Argon, Is Mediated by Inhibition at the N-Methyl-D-Aspartate Receptor Glycine Site. Anesthesiology 2013, 119(5):1137-1148.

46. Ryang YM, et al: Neuroprotective effects of argon in an in vivo model of transient middle cerebral artery occlusion in rats. Crit Care Med 2011, 39(6):1448-53

47. Zhuang $L$, et al: The protective profile of argon, helium, and xenon in a model of neonatal asphyxia in rats. Crit Care Med 2012, 40(6):1724-30.

48. Neumar RW, et al: Post-cardiac arrest syndrome: epidemiology, pathophysiology, treatment, and prognostication. A consensus statement from the International Liaison Committee on Resuscitation (American Heart Association, Australian and New Zealand Council on Resuscitation, European Resuscitation Council, Heart and Stroke Foundation of Canada, InterAmerican Heart Foundation, Resuscitation Council of Asia, and the Resuscitation Council of Southern Africa); the American Heart Association Emergency Cardiovascular Care Committee; the Council on Cardiovascular Surgery and Anesthesia; the Council on Cardiopulmonary, Perioperative, and Critical Care; the Council on Clinical Cardiology; and the Stroke Council. Circulation 2008, 118(23):2452-83.

49. Brucken $A$, et al: Argon reduces neurohistopathological damage and preserves functional recovery after cardiac arrest in rats. $\mathrm{Br} J$ Anaesth 2013, 110(suppl 1):i106-i112.

50. Ristagno $\mathrm{G}$, et al: Post-resuscitation treatment with argon improves early neurological recovery in a porcine model of cardiac arrest. Shock 2013, 41(1):72-78.

51. David HN, et al: Ex vivo and in vivo neuroprotection induced by argon when given after an excitotoxic or ischemic insult. PLoS One 2012, 7(2):e30934

52. Yang XR, et al: Involvement of MAPK pathways in NMDA-induced apoptosis of rat cortical neurons. Sheng Li Xue Bao 2012, 64(6):609-16.

53. Jin J, et al: The blockade of NMDA receptor ion channels by ketamine is enhanced in developing rat cortical neurons. Neurosci Lett 2013, 539:11-5.

54. Ali Shah $\mathrm{S}$, et al: Anthocyanins protect against ethanol-induced neuronal apoptosis via GABA receptors intracellular signaling in prenatal Rat hippocampal neurons. Mol Neurobiol 2013, 48(1):257-269.

55. Yang L, Sonner JM: Anesthetic-like modulation of receptor function by surfactants: a test of the interfacial theory of anesthesia. Anesth Analg 2008, 107(3):868-74.

56. Mihic SJ, et al: Sites of alcohol and volatile anaesthetic action on GABA (A) and glycine receptors. Nature 1997, 389(6649):385-9.

57. Randall RD, Thayer SA: Glutamate-induced calcium transient triggers delayed calcium overload and neurotoxicity in rat hippocampal neurons. J Neurosci 1992, 12(5):1882-95.

58. Choi DW, Koh JY, Peters S: Pharmacology of glutamate neurotoxicity in cortical cell culture: attenuation by NMDA antagonists. J Neurosci 1988, 8(1):185-96.

59. Yeganeh F, et al: Neuroprotective effects of NMDA and group I metabotropic glutamate receptor antagonists against neurodegeneration induced by homocysteine in rat hippocampus: in vivo study. J Mol Neurosci 2013, 50(3):551-7.

60. Lipton P: Ischemic cell death in brain neurons. Physio/ Rev 1999 79(4):1431-568

61. Dirnagl U, ladecola C, Moskowitz MA: Pathobiology of ischaemic stroke: an integrated view. Trends Neurosci 1999, 22(9):391-7.

62. Panaretakis T, et al: Activation of Bak, Bax, and $\mathrm{BH} 3$-only proteins in the apoptotic response to doxorubicin. J Biol Chem 2002, 277(46):44317-26.

63. Li Y, Han F, Shi Y: Increased neuronal apoptosis in medial prefrontal cortex is accompanied with changes of $\mathrm{Bcl}-2$ and bax in a rat model of post-traumatic stress disorder. J Mol Neurosci 2013, 51(1):127-137.

64. Costa $C$, et al: Coactivation of GABA (A) and GABA (B) receptor results in neuroprotection during in vitro ischemia. Stroke 2004, 35(2):596-600.

65. Wei XW, et al: Neuroprotection of co-activation of GABA receptors by preventing caspase-3 denitrosylation in KA-induced seizures. Brain Res Bull 2012, 88(6):617-23.

66. Dai J, et al: Activations of GABAergic signaling, HSP70 and MAPK cascades are involved in baicalin's neuroprotection against gerbil global ischemia/reperfusion injury. Brain Res Bull 2013, 90:1-9.

67. Subramaniam S, Unsicker K: ERK and cell death: ERK1/2 in neuronal death. FEBS J 2010, 277(1):22-29.

68. Fahlenkamp AV, et al: The noble gas argon modifies extracellular signal-regulated kinase $1 / 2$ signaling in neurons and glial cells. Eur $J$ Pharmacol 2012, 674(2-3):104-11.

69. David HN, et al: Modulation by the noble gas argon of the catalytic and thrombolytic efficiency of tissue plasminogen activator. Naunyn Schmiedebergs Arch Pharmacol 2013, 386(1):91-5.

70. Irani $Y$, et al: Noble gas (argon and xenon)-saturated cold storage solutions reduce ischemia-reperfusion injury in a rat model of renal transplantation. Nephron Extra 2011, 1(1):272-82.

71. Pagel PS, et al: Noble gases without anesthetic properties protect myocardium against infarction by activating prosurvival signaling kinases and inhibiting mitochondrial permeability transition in vivo. Anesth Analg 2007, 105(3):562-9.

72. Spaggiari $\mathrm{S}$, et al: Antiapoptotic activity of argon and xenon. Cell Cycle 2013, 12(16):2636-42.

73. Miyazawa T, et al: Early experiences of haemostasis on brain tumour surgery with Argon Plasma Coagulation (APC). Acta Neurochir (Wien) 2000, 142(11):1247-51.

74. Smythe A, et al: The effect of argon plasma coagulation ablation on esophageal motility and chemoreceptor sensitivity in Barrett's esophagus patients. Dis Esophagus 2010, 23(6):445-50.

75. Goulet CJ, et al: In vivo evaluation of argon plasma coagulation in a porcine model. Gastrointest Endosc 2007, 65(3):457-62.

76. Min BH, et al: Feasibility and efficacy of argon plasma coagulation for early esophageal squamous cell neoplasia. Endoscopy 2013, 45(7):575-8.

77. Ahn JY, et al: Clinical outcomes of argon plasma coagulation for the treatment of gastric neoplasm. Surg Endosc 2013, 27(9):3146-3152

78. Riegel T, et al: Comparative experimental study of argon plasma and bipolar coagulation techniques. Acta Neurochir (Wien) 2006, 148(7):757-62. discussion 762-3.

doi:10.1186/2045-9912-4-3

Cite this article as: Nowrangi et al:: Argon gas: a potential

neuroprotectant and promising medical therapy. Medical Gas Research 2014 4:3. 